\title{
Preimplantation Genetic Testing for Kidney Disease-Related Genes: A Laboratory's Experience
}

\author{
Jessica L. Chaperon ${ }^{a}$ Nina M. Wemmer ${ }^{a} \quad$ Trudy A. McKanna $^{a}$ Dinah M. Clark ${ }^{a}$ \\ Maggie A. Westemeyer ${ }^{\mathrm{a}}$ Philippe Gauthier ${ }^{\mathrm{a}}$ Yan Bai ${ }^{\mathrm{a}}$ Jessica M. Coleman ${ }^{\mathrm{b}}$

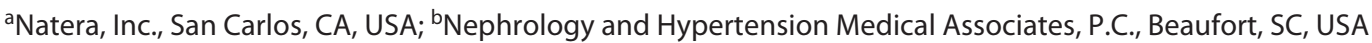

\section{Keywords}

Preimplantation genetic testing - Chronic kidney disease .

Monogenic kidney disease

\begin{abstract}
Introduction: Recent literature highlights the clinical utility of genetic testing for patients with kidney disease. Genetic testing provides significant benefits for reproductive risk counseling, including the option of in vitro fertilization with preimplantation genetic testing for monogenic disease (PGT-M). PGT-M allows for a significant reduction in risk for a pregnancy affected with the familial disease. We aim to summarize our experience with PGT-M for genes with kidney involvement as either a primary or secondary feature of the disease. Methods: All PGT-M tests performed by the reference laboratory between September 2010 and July 2020 were reviewed for clinical indication and cases for which the disease tested included a renal component. Each patient referred for PGT-M had an existing molecular genetic diagnosis themselves or in their family. Frequency of each condition, gene, inheritance pattern, and year over year increase in referral cases was analyzed. Results: In the study cohort, the most common disease targeted was autosomal dominant polycystic kidney disease, caused by pathogenic vari-
\end{abstract}

karger@karger.com www.karger.com/ajn

Karger $\stackrel{\text { ' }}{5}$

GOPEN ACCESS
C 2021 The Author(s).

Published by S. Karger AG, Basel

This is an Open Access article licensed under the Creative Commons Attribution-NonCommercial-4.0 International License (CC BY-NC) (http://www.karger.com/Services/OpenAccessLicense), applicable to the online version of the article only. Usage and distribution for commercial purposes requires written permission. ants in the PKD1 or PKD2 genes, which accounted for $16.5 \%$ (64/389) of cases. The 5 most common referral indications accounted for $51.9 \%(202 / 389)$ of the cases. Autosomal recessive inheritance accounted for $52.0 \%(26 / 50)$ of conditions for which PGT-M was performed. The number of PGT-M tests performed for conditions that included either primary or secondary kidney disease increased from 5 cases in 2010 to 47 cases in the 2020 study period. Discussion/Conclusion: These data suggest that the pursuit of PGT-M by couples at risk for passing on conditions with a kidney component is common and has significantly increased since 2010. With this rising trend of patients undergoing PGT-M and the prerequisite of molecular genetic confirmation in the PGT-M process, this study underscores the importance of the reproductive component to a molecular genetic diagnosis for patients with kidney disease, especially as the accessibility of genetic testing and utilization by nephrologists grows.

(C) 2021 The Author(s).

Published by S. Karger AG, Basel

\section{Introduction}

Approximately 1 in 7 US adults is estimated to have chronic kidney disease (CKD), which equates to over 30 million adults being affected by the condition [1]. The 
etiology of CKD may be genetic, metabolic, developmental, degenerative, related to infectious, toxic, or traumatic events, or is often unknown [2]. Given the significant morbidity and mortality associated with the disease, the Executive Branch of the US government announced a set of initiatives in 2019 collectively titled Advancing American Kidney Health [3]. This initiative seeks to reduce the risk of kidney failure, improve access to and quality of individual-centered treatment options, and increase access to kidney transplants [3].

For patients with a genetic etiology for their kidney disease, individual-centered treatment options enable tailored counseling, risk assessment, and treatment. In the past few decades, significant technical progress has been made in genetic testing that allowed for an increase in the ability to uncover a molecular genetic diagnosis for kidney disease [4]. A recent study demonstrated the diagnostic yield of genetic testing in adult patients with CKD to be about $10 \%$, similar to the diagnostic yield of hereditary cancer testing, which, in the last decade, has become widely accepted by the National Comprehensive Cancer Network $[5,6]$. The benefits of determining the molecular cause of kidney disease are many; it allows for confirmation or reclassification of a clinical diagnosis, enabling a patient-centric treatment; it provides diagnosis for kidney disease with unknown etiology, identifies the risk of extrarenal disease associated with the primary condition, and may allow the patient to enroll in appropriate clinical trials. Importantly, early identification of a genetic etiology permits family risk counseling and planning. Notably, a significant proportion of individuals with kidney disease have a family history of the condition, and thus genetic testing often affects multiple family members including future offspring of an affected individual [7]. A recent study by Snoek et al. [8] that presented experience in the Netherlands with preimplantation genetic testing for monogenic kidney disease (PGT-M) underscored the benefit of this reproductive option for patients with kidney disease.

PGT-M is one reproductive option available to individuals who carry a disease-causing genetic variant. It enables selection and transfer of embryos that are determined to not carry the variant(s) of concern, thereby greatly reducing the chance of having a pregnancy affected with the condition, typically by $>95 \%$. PGT-M is performed as part of an in vitro fertilization (IVF) cycle and requires embryo biopsy which may occur at the cleavage stage (day 3 of development) with removal of 1-2 cells or more commonly at the blastocyst stage (day 5-7 of development) with removal of up to 10 cells [9]. While the IVF cycle and biopsy procedure occur at an IVF clinic, the biopsy samples are typically sent to a PGT-M reference laboratory for testing. PGT-M test development is customized and is therefore unique to each individual, but generally requires confirmation of the disease-causing variant and determining which parental chromosome contains the variant. This is determined by identifying informative DNA markers, such as single-nucleotide polymorphisms (SNPs) or short tandem repeats surrounding the disease gene variant, and/or directly testing for the variant(s) in the biopsy samples by sequencing $[10,11]$. Test development requires DNA samples from the biological parents of the embryos and may require samples from other affected and/or unaffected relatives. Parent and relative samples are evaluated for the informative markers, and clinical information regarding their disease or carrier status is referenced to determine which DNA markers are linked to the familial mutation $[10,11]$.

Embryos are tested indirectly by linkage analysis of polymorphic markers and may also be tested directly by mutation analysis. Embryos that do not contain the mutation(s) are then preferentially selected for transfer. Chromosome abnormalities are common causes of implantation failure in IVF and are the most common cause of early pregnancy loss $[12,13]$. The frequency of chromosome abnormalities in embryos increases with maternal age, but aneuploidy is relatively common across all age groups in embryo samples $[14,15]$. Some platforms allow for simultaneous preimplantation genetic testing for aneuploidy (PGT-A) with PGT-M [16], thereby improving implantation and pregnancy rates while decreasing miscarriage rates $[17,18]$. In this study, we present a commercial laboratory's experience with PGT-M for conditions related to primary or secondary kidney disease with concurrent PGT-A.

\section{Materials and Methods}

Over a 10-year period, patients were referred by their IVF centers to a reference laboratory for PGT-M with concurrent 24-chromosome PGT-A using an informatics-based SNP microarray technology (Parental Support ${ }^{\mathrm{TM}}$ ). Each patient referred for PGT-M had an existing molecular genetic diagnosis. DNA from both parents and from biopsied embryo cells was amplified and genotyped using a 300,000-probe SNP microarray (HumanCyroSNP-12; Illumina, San Diego, CA, USA) as described $[19,20]$. Parental SNP genotypes were used to haplotype the embryo, and approximately 35 informative, neighboring SNPs were used to determine which haplotype, and thus which embryo, contained the target mutation. The same SNP markers were included on the array for all cases; however, the number of informative SNP markers surrounding the disease gene mutation varied based on the location of the vari- 
Table 1. Referral indications for PGT-M with a kidney component

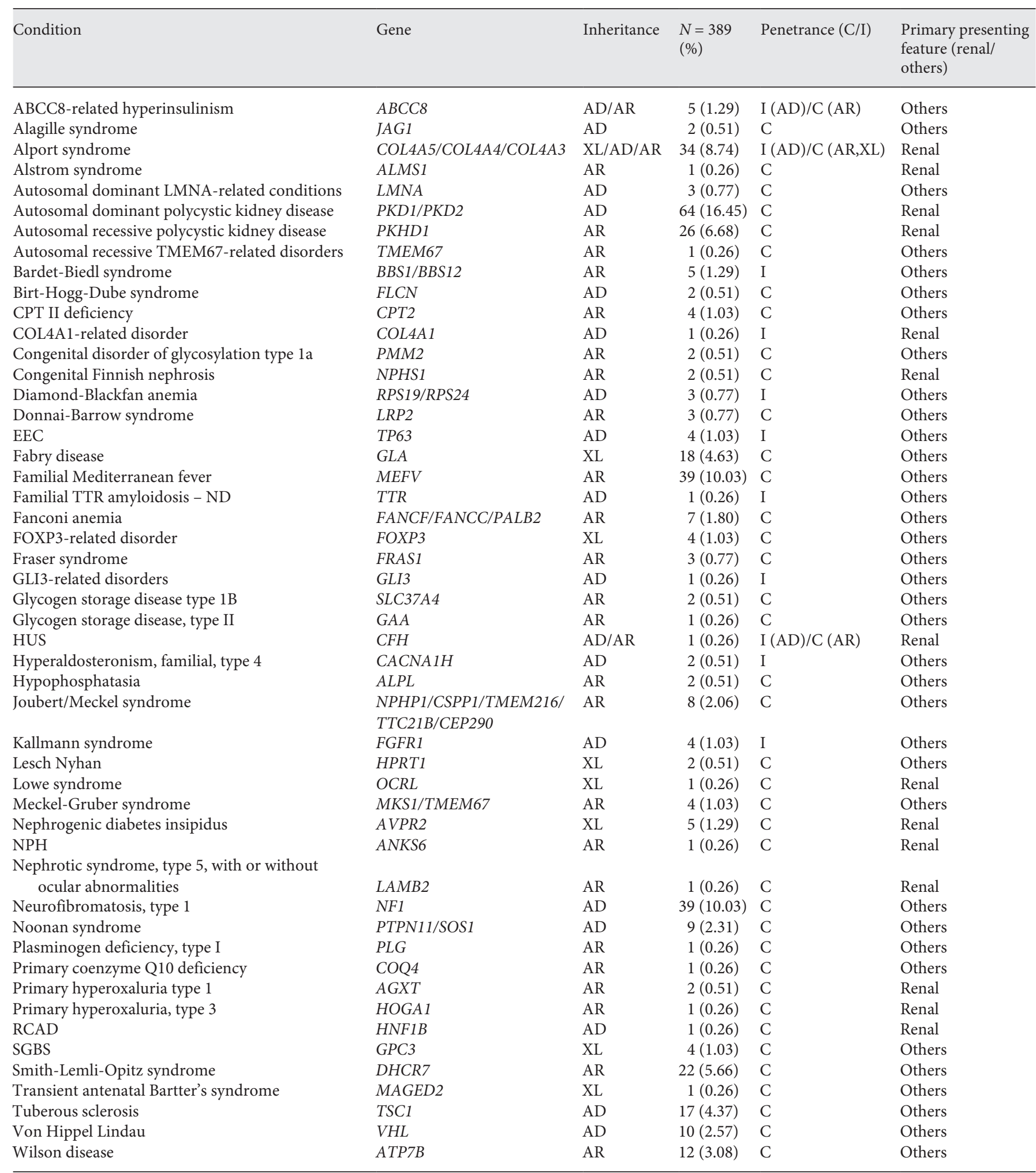

$\mathrm{AD}$, autosomal dominant; $\mathrm{AR}$, autosomal recessive; XL, X-linked; C, complete penetrance; I, incomplete penetrance; SGBS, Simpson-Golabi-Behmel syndrome; RCAD, renal cysts and diabetes syndrome; NPH, nephronophthisis; HUS, hemolytic uremic syndrome; EEC, ectrodactyly-ED-clefting syndrome; CPT II, carnitine palmitoyltransferase II. 
ation and parental genotypes. In cases where relative samples were either not available or not informative in determining which haplotype contained the mutation, custom primers were designed to directly detect the mutation in the embryo sample. Mutation and aneuploidy results were reported back to the referring IVF centers. The number of embryos that were suitable for transfer based on their chromosomally normal (euploid) and unaffected status was recorded for each cycle. No live birth or follow-up data were collected for the purpose of this analysis.

A retrospective analysis was performed on all PGT-M tests performed at a reference laboratory between September 2010 and July 2020, along with concurrent PGT-A tests. Patient consent was not necessary, and ethical approval was not required, as this analysis included nonclinical deidentified data from the commercial ordering history. Here, we report the number of cases that pursued PGT-M for conditions with either a primary or secondary kidney component and the breakdown of the conditions tested. The number of cases reflects tests run for a given condition and may include multiple cycles for the same patient.

\section{Results}

In the 10-year study period, the reference laboratory analyzed a total of 3,287 cases for PGT-M, of which $11.8 \%$ $(389 / 3,287)$ were tested for conditions with kidney involvement and were referred from 98 different IVF centers. Six of the 389 cases used an egg donor. Of the cases that did not use an egg donor, the mean maternal age was 34 years (range $23-45$ years).

In the 389 cases where PGT-M was performed, 2,578 embryo biopsy samples were analyzed. Of the 389 cases, $5 \%(19 / 389)$ underwent day 3 biopsy and the remaining 95\% underwent day 5-7 biopsy. In total, 73\% (284/389) of cases tested had at least 1 unaffected and chromosomally normal embryo identified for transfer. For the remaining $27 \%$ (105/389) of cases, all of the tested samples were found to be affected with the single gene disorder of concern and/or had aneuploid chromosome results. During the study period, $4.6 \%$ of embryos had no results reported due to insufficient embryo DNA or uninformative results.

A total of 65 genes associated with 50 different conditions that included a kidney component were tested (Table 1). Kidney disease was the primary presenting feature for $28 \%(14 / 50)$ of the conditions tested, while the other $72 \%$ were systemic diseases with the potential for renal manifestations. Of the conditions tested, 52\% (26/50) were autosomal recessive, $32 \%(16 / 50)$ were autosomal dominant, 14\% (7/50) were X-linked, and 1 condition, Alport syndrome, has varying inheritance depending on the gene involved (shown in Fig. 1). In the cohort, the most common referral indications for PGT-M were auto-

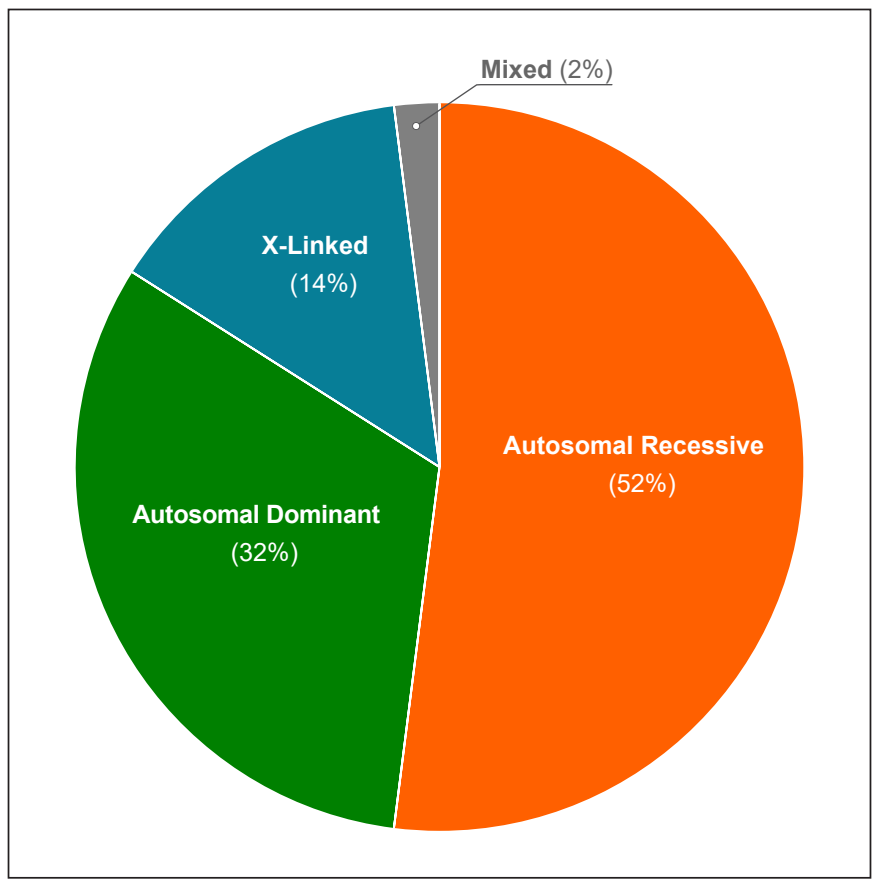

Fig. 1. Distribution of inheritance patterns: pie chart showing percentage of examined genes, based on pattern of inheritance: autosomal dominant, autosomal recessive, or X-linked.

somal dominant polycystic kidney disease (ADPKD) (16.5\%), familial Mediterranean fever (10.03\%), neurofibromatosis, type 1 (10.03\%), Alport syndrome (8.74\%), autosomal recessive polycystic kidney disease (6.68\%), Smith-Lemli-Opitz syndrome (5.66\%), Fabry disease $(4.63 \%)$, tuberous sclerosis $(4.37 \%)$, Wilson disease (3.08\%), and Von Hippel Lindau (2.57\%) (Table 1). The remainder of the conditions comprised $27.76 \%$ of the total cases with 21 of these conditions having a single referral (shown in Fig. 2). Of the ADPKD cases, 81\% (52/64) were referred for analysis of a variant in the $P K D 1$ gene, and the remaining $19 \%(12 / 64)$ were referred for analysis of a variant in the PKD2 gene. Of the Alport syndrome cases, $60.6 \%(20 / 33)$ were referred for analysis of variants in the COL $4 A 5$ gene, followed by $21.2 \%$ (7/33) for analysis of variants in the COL4A4 gene and $18.2 \%(6 / 33)$ for analysis of variants in the COL $4 A 3$ gene.

Of the 50 conditions tested, $78 \%$ have complete penetrance, $16 \%$ have incomplete penetrance, and for $6 \%$ of the conditions, penetrance is reduced in the dominant form of the condition but fully penetrant for the recessive form. For example, a single variant in the COL4A5 genes is expected to be completely penetrant for Alport syndrome, while a single variant in either the COL $4 A 3$ or 


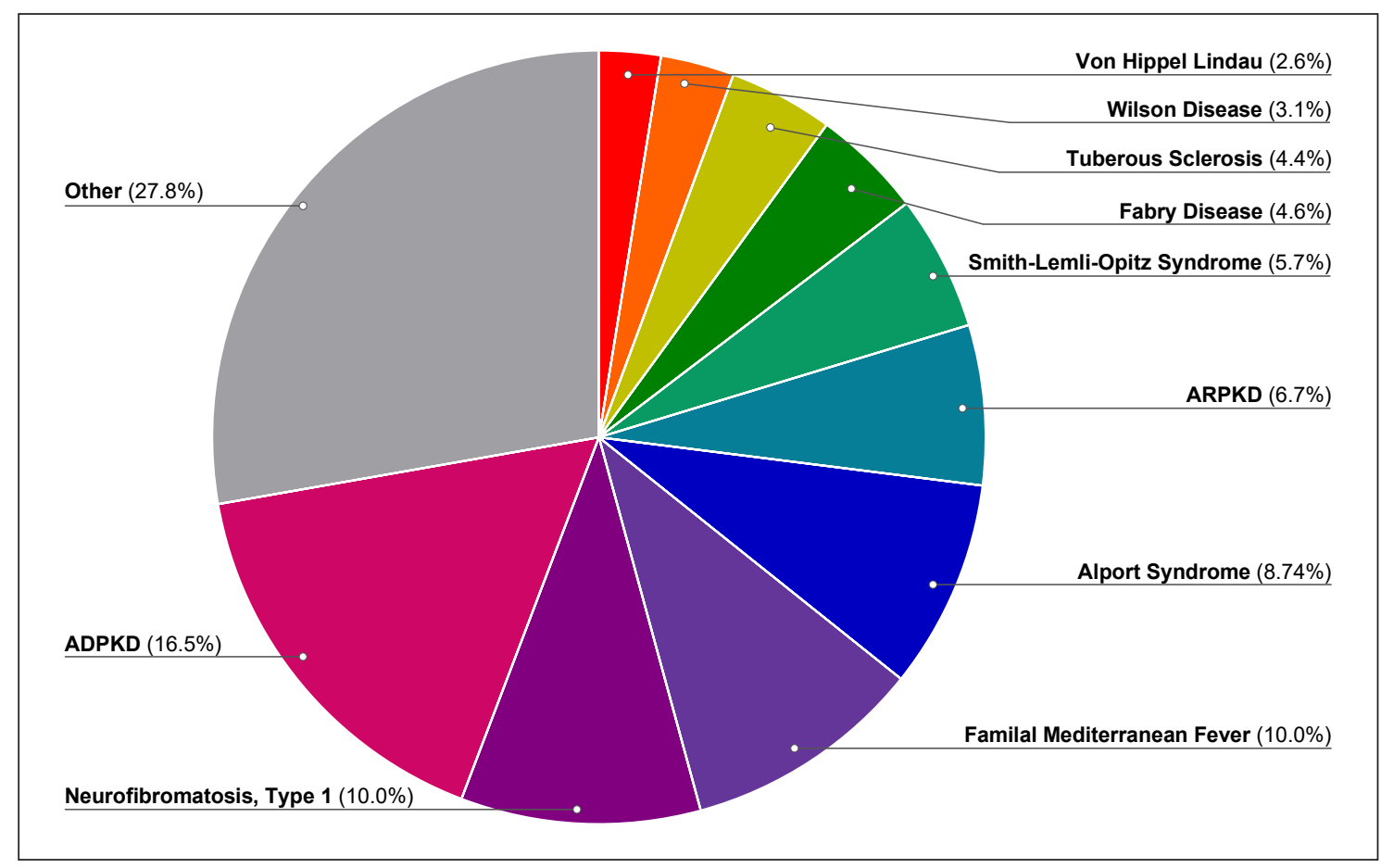

Fig. 2. Proportion of total cases accounted for by top 10 diagnoses: pie chart showing the most common clinical indications for PGT-M associated with primary or secondary kidney disease in the cohort. PGT-M, preimplantation genetic testing for monogenic disease; ADPKD, autosomal dominant polycystic kidney disease; ARPKD, autosomal recessive polycystic kidney disease.

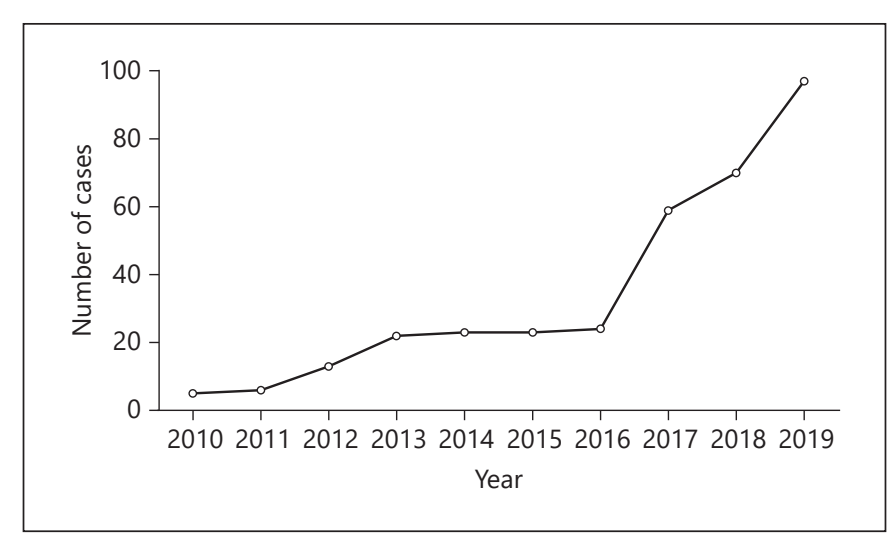

Fig. 3. PGT-M cases with a kidney component by year: the increased number of cases pursuing PGT-M for conditions with a kidney component is represented, from 2010 to 2019. PGT-M, preimplantation genetic testing for monogenic disease.

COL4A4 gene has incomplete penetrance. Of note, the inheritance pattern for both $A B C C 8$-related hyperinsulinism and hemolytic uremic syndrome varies based on the variant in the gene. In this dataset, the only variants involved were autosomal dominant. The number of cases tested for conditions related to kidney disease was examined year over year (2010 through 2019), for years with complete data collected. The number of cases tested per year remained relatively consistent from 2013 to 2016 and increased sharply from 2017 to 2019 (shown in Fig. 3).

\section{Discussion/Conclusion}

The number of kidney-related PGT-M tests performed by our commercial laboratory over the past 10 years underscores the importance of molecular genetic testing for patients with kidney disease and the importance of comprehensive counseling regarding reproductive options for patients of child-bearing age. While not all conditions included in this analysis primarily affect the kidney, patients with these diseases may present for care in general nephrology and therefore were considered relevant to our study. Of the total number of PGT-M cases referred to the lab during the study period, $11.8 \%$ were for conditions re- 
lated to kidney disease. While a clinical diagnosis may be sufficient for treatment and management of a patient's kidney disease, identification of the disease-causing genetic variant is required for PGT-M. Additionally, PGT$M$ requires personalized test development, which includes obtaining DNA samples from the patient and partner, and in many cases, from the patient or partner's parents and/ or their children as well. Even if the patient in question may be beyond reproductive age, a molecular diagnosis can be useful for a patient's children or other first-degree relatives who may benefit from the option of PGT-M. Molecular diagnosis is also critical for cases in which a specific disease can have multiple inheritance patterns, such as Alport syndrome, which can have X-linked, autosomal dominant, or autosomal recessive inheritance depending on the gene and variant(s) involved. While a clinical diagnosis for Alport syndrome is possible, identifying which relatives are at risk for the disease is dependent upon the molecular diagnosis. Knowledge of the inheritance pattern and risk of inheritance by an individual's offspring may impact their choices regarding reproductive options.

The availability of PGT-M has an important clinical impact in general nephrology practice. In the spectrum of genetic kidney diseases, specifically the most common disease state, polycystic kidney disease, nephrologists frequently guide complex patient decisions. These complex decisions include family planning, establishing life insurance (including presymptomatic diagnosis for at-risk possibly affected family members), and emotional support. Given that nephrologists are already accustomed to guiding complex decision-making for their patients, it seems logical that the availability of PGT-M will further advance these conversations. While the anticipation is that the conversations for PGT-M will be held between the patients and their reproductive specialists, we have to conclude that initial conversations will start with the patient's nephrologist. Therefore, it encourages nephrologists to understand the principles and advantages that PGT-M confers in family planning services as well as, potentially, in their own treatment and care options. This option provides hope for patients who do not desire to transmit a genetic condition where previously there were limited, or no, options available. Heritable kidney diseases are the fourth most common cause of ESRD in the USA, thus highlighting the potential ability of PGT-M to minimize kidney disease risk in offspring and therefore prevent the need for medical care associated with disease, which can be life altering for affected families.

Identification of disease-causing variant(s) in an individual is not only necessary for PGT-M but also may deter-

Preimplantation Genetic Testing for

Kidney Disease mine whether or not PGT-M is an option for the patient or family. Certain types of variants may require DNA samples from relatives and/or documentation of a relative's variant status in order to develop a PGT-M test. For example, mutations in PKD1 typically cannot be tested directly in embryos due to the presence of a pseudogene which complicates primer design. For these patients, the availability of relative samples is crucial for PGT-M test development. Genetic counselors play an important role in explaining the feasibility and options for PGT-M given the specific variant(s) detected and navigating family dynamics.

In this cohort, $30.8 \%$ of cycles were screened for autosomal dominant genes, $53.8 \%$ for autosomal recessive genes, $13.5 \%$ for X-linked genes, and $1.8 \%$ for genes with both autosomal dominant and autosomal recessive inheritance. In these scenarios, the risk of an affected embryo can be as high as $50 \%$. PGT-M enables significant reduction of that risk, with reported misdiagnosis rates of $<1 \%$ [21]. Reasons for misdiagnosis include technical errors, such as allele-drop-out or contamination, and human error, such as sample mislabeling or incorrect embryo transfer [16]. 284/389 cases analyzed had at least 1 embryo that was euploid and unaffected for disease, meaning that in $73 \%$ of cases, PGT-M allowed for a significant risk reduction in the transmission of a heritable disease being passed onto future generations.

Of note, some embryos may have been negative for the familial mutation but had chromosomal aneuploidy and were therefore not candidates for transfer. Additionally, 2 cases tested for $>1$ Mendelian disorder (1 kidney related and 1 nonkidney related) may have had embryos excluded from transfer due to results for the nonkidney-related condition.

A limitation of this study is that longitudinal follow-up information was not collected, preventing us from analyzing pregnancy outcomes and the live birth rate resulting from PGT-M. However, there were no reported PGT$\mathrm{M}$ misdiagnoses among our cohort.

The increase in PGT-M referrals for conditions related to kidney disease observed between 2016 and 2019 may be related to numerous factors including increases in the availability of diagnostic molecular testing for kidney disease, utilization of preconception expanded carrier screening, expanding insurance coverage for fertility, and awareness of the option of PGT-M among couples at risk for pregnancies affected by Mendelian disease. In conclusion, awareness of PGT-M as a reproductive option for couples amongst the nephrology community is critical as the need for decision-making support for patients and appropriate referrals to reproductive specialists increases. 


\section{Acknowledgments}

The authors would like to acknowledge Dr. Neera Dahl from Yale University School of Medicine for her review and feedback on the manuscript and the editorial support provided by the scientific communications team at Natera, Inc., San Carlos.

\section{Statement of Ethics}

No ethical approval was required for this study. Patient consent was not necessary as this analysis includes nonclinical deidentified data from the commercial ordering history.

\section{Conflict of Interest Statement}

J. Chaperon is an employee of Natera, Inc., with the option to hold stock in the company. D. Clark is an employee of Natera, Inc., with the option to hold stock in the company. N. Wemmer is an employee of Natera, Inc., with the option to hold stock in the company. T. McKanna is an employee of Natera, Inc., with the option to hold stock in the company. M. Westemeyer is an employee of Natera, Inc., with the option to hold stock in the company. P.
Gauthier is an employee of Natera, Inc., with the option to hold stock in the company. Y. Bai is an employee of Natera, Inc., with the option to hold stock in the company. Jessica Coleman serves as a paid advisor for Natera, Inc.

\section{Funding Sources}

This study received no funding.

\section{Author Contributions}

J. Chaperon was involved in conceptualization; data curation; formal analysis; investigation; writing - original draft, review, and editing. D. Clark was involved in conceptualization; data curation; formal analysis; investigation; writing - original draft, review, and editing. N. Wemmer was involved in conceptualization; data curation; formal analysis; investigation; writing - original draft, review, and editing. T. McKanna was involved in conceptualization and writing - review and editing. M. Westemeyer was involved in writing - review and editing. P. Gauthier was involved in writing - review and editing. Y. Bai was involved in writing - review and editing. J. Coleman was involved in writing - review and editing.

\section{References}

1 Services USDoHaH. Organ procurement and transplantation network; 2020. Available from: https://optn.transplant.hrsa.gov/data/.

2 Connaughton DM, Hildebrandt F. Personalized medicine in chronic kidney disease by detection of monogenic mutations. Nephrol Dial Transplant. 2020 Mar 1;35(3):390-7.

3 Mehrotra R. Advancing American kidney health: an introduction. Clin $\mathrm{J}$ Am Soc Nephrol. 2019;14(12):1788-8.

4 Groopman EE, Rasouly HM, Gharavi AG. Genomic medicine for kidney disease. Nat Rev Nephrol. 2018;14(2):83-104.

5 Groopman EE, Marasa M, Cameron-Christie S, Petrovski S, Aggarwal VS, Milo-Rasouly H, et al. Diagnostic utility of exome sequencing for kidney disease. N Engl J Med. 2018;380(2): $142-51$.

6 National Comprehensive Cancer Network. NCCN clinical practice guidelines in oncology: breast, ovarian and pancreatic: National Comprehensive Cancer Institute; 2020.

7 Verma M. Genetic testing is beneficial to the entire family. Clin J Am Soc Nephrol. 2020; 15(9):1224-4.

8 Snoek R, Stokman MF, Lichtenbelt KD, van Tilborg TC, Simcox CE, Paulussen ADC, et al. Preimplantation genetic testing for monogenic kidney disease. Clin J Am Soc Nephrol. 2020;15(9):1279-86.

9 Consortium EP, Kokkali G, Coticchio G, Bronet F, Celebi C, Cimadomo D, et al. ESHRE PGT Consortium and SIG Embryology good practice recommendations for polar body and embryo biopsy for PGT. Hum Reprod Open. 2020;2020(3): hoaa020.

10 Gutiérrez-Mateo C, Sánchez-García JF, Fischer J, Tormasi S, Cohen J, Munné S, et al. Preimplantation genetic diagnosis of singlegene disorders: experience with more than 200 cycles conducted by a reference laboratory in the United States. Fertil Steril. 2009; 92(5):1544-56.

11 Handyside AH, Harton GL, Mariani B, Thornhill AR, Affara N, Shaw MA, et al. Karyomapping: a universal method for genome wide analysis of genetic disease based on mapping crossovers between parental haplotypes. J Med Genet. 2010 Oct;47(10):651-8.

12 Gianaroli L, Magli MC, Ferraretti AP, Fiorentino A, Garrisi J, Munné S. Preimplantation genetic diagnosis increases the implantation rate in human in vitro fertilization by avoiding the transfer of chromosomally abnormal embryos. Fertil Steril. 1997 Dec;68(6):1128-31.

13 Menasha J, Levy B, Hirschhorn K, Kardon NB. Incidence and spectrum of chromosome abnormalities in spontaneous abortions: new insights from a 12-year study. Genet Med. 2005 Apr;7(4):251-63.

14 Rabinowitz M, Ryan A, Gemelos G, Hill M, Baner J, Cinnioglu C, et al. Origins and rates of aneuploidy in human blastomeres. Fertil Steril. 2012;97(2):395-401.

15 Franasiak JM, Forman EJ, Hong KH, Werner MD, Upham KM, Treff NR, et al. Aneuploidy across individual chromosomes at the embryonic level in trophectoderm biopsies: changes with patient age and chromosome structure. J Assist Reprod Genet. 2014;31(11):1501-9.

16 De Rycke M, Berckmoes V. Preimplantation genetic testing for monogenic disorders. Genes. 2020;11(8):871.

17 Rechitsky S, Pakhalchuk T, San Ramos G, Goodman A, Zlatopolsky Z, Kuliev A. First systematic experience of preimplantation genetic diagnosis for single-gene disorders, and/ or preimplantation human leukocyte antigen typing, combined with 24-chromosome aneuploidy testing. Fertil Steril. 2015 Feb; 103(2):503-12.

18 Hou W, Xu Y, Li R, Song J, Wang J, Zeng Y, et al. Role of aneuploidy screening in preimplantation genetic testing for monogenic diseases in young women. Fertil Steril. 2019 May; 111(5):928-35.

19 Johnson DS, Gemelos G, Baner J, Ryan A, Cinnioglu $\mathrm{C}$, Banjevic $\mathrm{M}$, et al. Preclinical validation of a microarray method for full molecular karyotyping of blastomeres in a 24-h protocol. Hum Reprod. 2010 Apr;25(4):1066-75.

20 Simon AL, Kiehl M, Fischer E, Proctor JG, Bush MR, Givens C, et al. Pregnancy outcomes from more than 1,800 in vitro fertilization cycles with the use of 24-chromosome single-nucleotide polymorphism-based preimplantation genetic testing for aneuploidy. Fertil Steril. 2018 Jul 1;110(1):113-21.

21 Wilton L, Thornhill A, Traeger-Synodinos J, Sermon KD, Harper JC. The causes of misdiagnosis and adverse outcomes in PGD. Hum Reprod. 2009;24(5):1221-8. 